\title{
SCIDOC
}

\author{
International Journal of Dentistry and Oral Science (IJDOS) \\ ISSN: 2377-8075
}

\section{Root Canal Morphology of the Mesiobuccal Root of Maxillary First Molars: CBCT Study}

Research Article

M.P. Induja ${ }^{1}$, Anjaneyulu K² M.P. Santhosh $\mathrm{Kumar}^{3^{*}}$

${ }^{1}$ Department of Oral and Maxillofacial Surgery, Saveetha Dental College and Hospital, Saveetha University, India.

${ }^{2}$ Reader, Department of Conservative Dentistry and Endodontics, Saveetha Dental College and Hospital, Saveetha University, India.

${ }^{3}$ Reader, Department of Oral and Maxillofacial Surgery, Saveetha Dental College and Hospital, Saveetha Institute of Medical and Technical Sciences (SIMATS), Saveetha University 162, Poonamallee High Road, Velappanchavadi, Chennai 600077 Tamil Nadu, India.

\section{Abstract}

Objective: Understanding tooth anatomy is crucial for effective endodontic treatment. This study investigated the roots and root canal morphologyof the mesiobuccal root of the maxillary first molars using cone-beam computed tomographic (CBCT) imaging.

Methods: A total of 18 teeth with intact roots were collected.Mesiobuccal [MB] root was sectioned completely with diamond discsandMB2canals was located using size 10file and the orifice was enlarged using slow speed hand piece and bur.

Results: Overall $44 \%$ of tooth had type 2 vertucci classification, $33.3 \%$ of tooth had type 4 Vertucci classification and $11.1 \%$ of tooth had type 1 and 6 vertucci classification.

Conclusion: Incidence of MB2 canals was $88.9 \%$. Outof 18 teeth with intact roots, 16 teeth had MB2 canals.Type IIVertucci classification was seen in most of the teeth, where MB2 canals were present.

Keywords: Maxillary First Molar; Mesiobuccal Root; Micro-Computed Tomography; Root Canal Anatomy; Vertucci Classification.

\section{Introduction}

The maxillary first molar is the earliest permanent tooth that appear in the oral cavity and that makes it vulnerable to caries and endodontic treatment. It has three roots and four canals in the most common form. Maxillary molar hasthe most complex root and root canal anatomy, and is possibly the most treated posterior tooth. The mesiobuccal root of maxillary molars has generated more research and clinical investigation than any other root [1].

The success of endodontic treatment is based on cleaning and shaping of the root canals. The root canals have complex morphology and wide individual variations. The objective of this study was to analyze root canals morphology and existence of extra canals in maxillary molars.Any existing root canals that remain undetected by the operator during the entire course of endodontic treatment are a major threat to the success of treatment. The first molar is the earliest permanent tooth to appear in the oral cavity exposing it for decay and in need of endodontic treatment [2]. A number of studies have been publishedregarding maxillary first molar root canals morphology using various ethnic groups, methods, and approaches.

There are several methods to identify root canal morphology such as canal staining and tooth clearing, conventional radiograph, digital radiographic techniques,radiographic assessment enhanced with contrast media, cone beam computed tomography (CBCT) techniques and modified canal staining and clearing. The CBCT is verified as a reliable tool for studying internal anatomy of tooth [3].

$\mathrm{CBCT}$ is a technique that uses a specific beam to produce three dimensional images to reveal anatomic details precisely. The key advantages of using CBCT are that it is non-invasive and per-

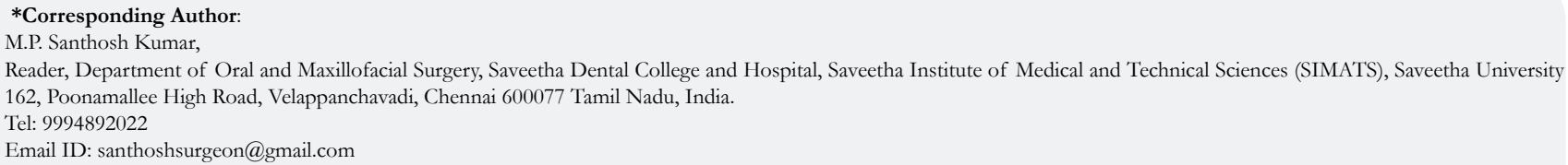

Citation: M.P. Induja, Anjaneyulu K, M.P. Santhosh Kumar. Root Canal Morphology of the Mesiobuccal Root of Maxillary First Molars: CBCT Study. Int J Dentistry Oral Sci. 2021;08(5):2416-2419. doi: http://dx.doi.org/10.19070/2377-8075-21000475

Copyright: M.P. Santhosh Kumar $^{\circ}$ 2021. This is an open-access article distributed under the terms of the Creative Commons Attribution License, which permits unrestricted use, distribution and reproduction in any medium, provided the original author and source are credited. 
mits 3-D reconstruction of the root canals. CBCT has the ability to identify the root canal system precisely,which is considered as better than other techniques to understand the root and canal systems.Regular computed tomography uses fan shaped beams, whereas CBCT uses cone shaped beams $[4,5]$. The aim of this study was to analyse the number of root canals in the mesiobuccal root of the maxillary first molars using Vertucci classification.

\section{Material and Methods}

A total of 18teeth with intact roots were collected in this study and it was arranged in a proper manner(Figure A). First, the crown part of the maxillary first molars was sectioned (Figure B) and then the Mesio buccal root was sectioned completely with diamond discs(Figure C). MB2 canals was located using size 10file; orifice was enlarged using the slow speed handpiece and bur,as it allows improved access for the flow of irrigant solution within the canal. It is important because the majority of bacteria in an infected root canal are located in coronal region. All maxillary molar images were analyzed carefully for roots (number), configuration and number of canals. In order to classify the root canal morphology, Vertucci's classification was used as a reference. Cone beam computed tomography images were obtained from the department of Oral medicine and diagnosis,Saveetha Dental college and hospitals.

\section{Result}

Figure 1 shows Cone beam tomography images of mesiobuccal root of maxillary molar with Type 2 (2-1) Vertucci classification; Two canals joined into one canal. Figure 2 demonstrates Cone beam tomography images of mesiobuccal root of maxillary molar showing Type 6 (2-1-2) Vertucci classification; two Canals joined into one canal and redivided into two canals.
Figure3 shows Cone beam tomography images of mesiobuccal root of maxillary molars depicting type 2 (2-1)Vertucci classification. Figure 4 demonstrates Cone beam tomography images of mesiobuccal root of maxillary molars showing type1 (1-1) Vertucci classification.

Figure 5 presentsCone beam tomography images of mesiobuccal root of maxillary molars showing type 4 (2-2) Vertucci classification; two separate canals. Figure 6 depicts Cone beam tomography images of mesiobuccal root of maxillary molars showing type 2 (2-1) Vertucci classification; Two canals joined into one canal.

Figure7 showsCone beam tomography images of mesiobuccal root of maxillary molars with type 4 (2-2) Vertucci classification; two separate canals. Figure 8 represents Cone beam tomography images of mesiobuccal root of maxillary molars showing type 6 (2-1-2) Vertucci classification; two Canals joined into one canal and redivided into two canals. Figure 9 depicts Cone beam tomography images of mesiobuccal root of maxillary molars showing type 4 (2-2) Vertucci classification; two separate canals. Figure 10 demonstratesCone beam tomography images of mesiobuccal root of maxillary molars showing type1(1-1) Vertucci classification; single canal. Figure 11 presents Cone beam tomography images of mesiobuccal root of maxillary molars showing type 6 (21-2) Vertucci classification; two Canals joined into one canal and redivided into two canals. Figure 12 shows Cone beam tomography images of mesiobuccal root of maxillary molars with type 2 (2-1)Vertucci classification; Two canals joined into one canal.

Overall $44 \%$ of tooth had type 2 vertucci classification, $33.3 \%$ of tooth had type 4 Vertucci classification and $11.1 \%$ of tooth had type 1 and 6 vertucci classification (Table1).

Figure A. Orderly arrangement of teeth samples used in the study.

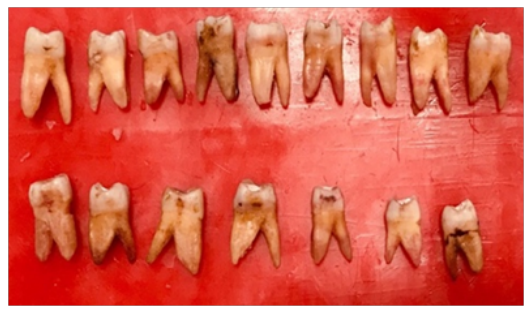

Figure B. Sectioned crowns of the maxillary first molars.

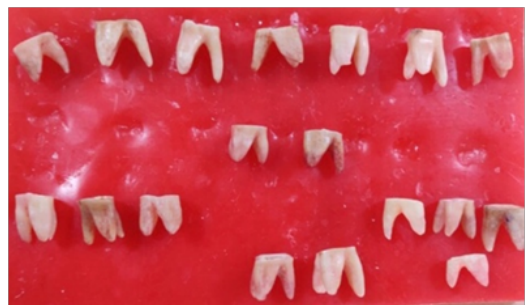

Figure C. Sectioned mesiobuccal root of the maxillary first molars.

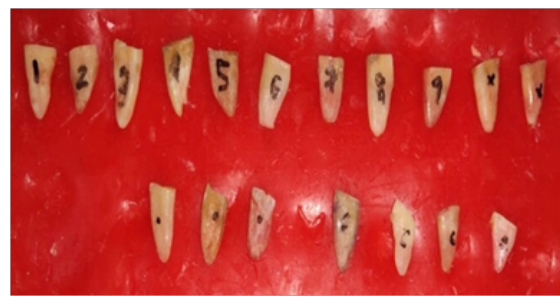


Figures 1-12. Represents the cone beam computed tomography images of mesiobuccal root of maxillary first molars.

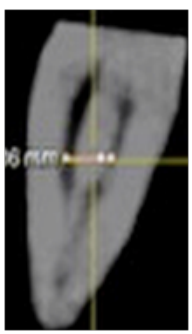

Fig 1.

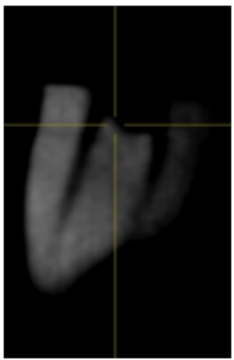

Fig 7.

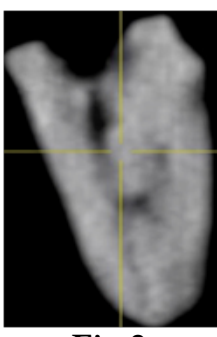

Fig 2.

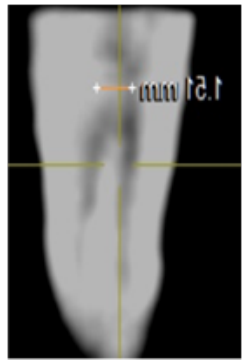

Fig 8.

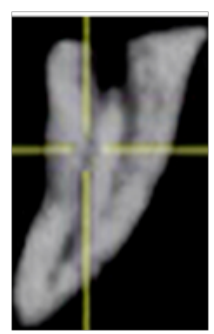

Fig 3.

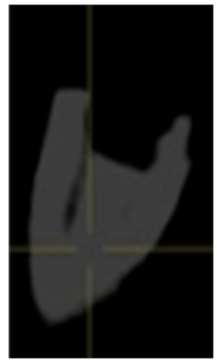

Fig 9.

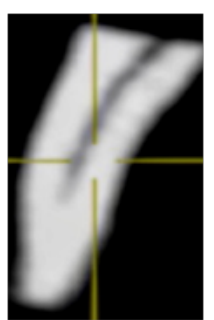

Fig 4.

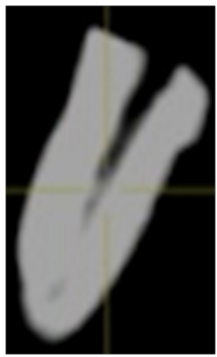

Fig 10.

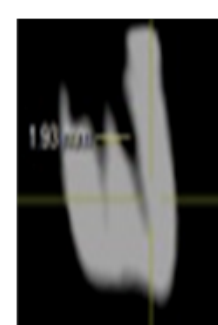

Fig 5.

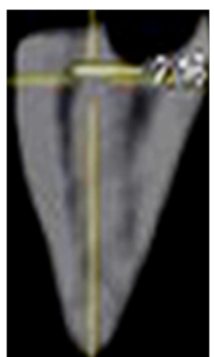

Fig 11.

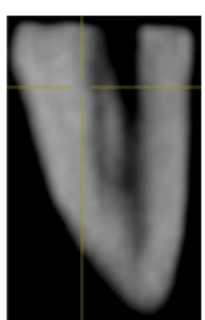

Fig 6.

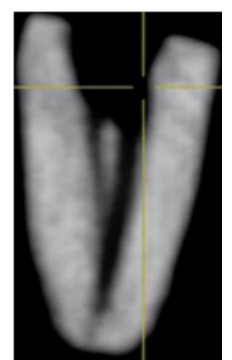

Fig 12.

Table 1.Vertucci classification of MB2 Canals.

\begin{tabular}{|c|c|c|}
\hline Vertucci & Classification & Percentage \\
\hline $1-1$ & Type I & $11.10 \%$ \\
\hline $2-1$ & Type II & $44.40 \%$ \\
\hline $2-2$ & Type IV & $33.30 \%$ \\
\hline $2-1-2$ & Type VI & $11.10 \%$ \\
\hline
\end{tabular}

\section{Discussion}

Successful endodontic therapy requires a significant knowledge of the anatomic features of root and root canal system. The lack of knowledge and missing a root canal are the most common reasons for failure in endodontic treatment. The significance of knowledge about the anatomic morphology of the maxillary molars and possible variations is vital for successful endodontic therapy and cannot be denied. The current study provides a detailed report on the morphology of root canals of maxillary $1 \mathrm{st}$ molars using CBCT. It is largely recognized that the most frequent pattern of the permanent maxillary 1 st molar is comprised of three separated roots. The mesiobuccal root is the main focus of morphological studies as the incidence of more than one canal is significantly high and a wide range of variations has been reported in the literature [6].

In 1969, Weine et al. provided the first clinical classification of more than one canal system in a single root and used the mesiobuccal root of the maxillary first molar as the type specimen [7]. Vertucci et al further developed a system for canal anatomy classification for any tooth that has a broad buccolingual diameter that may be more applicable for use in laboratory studies [8].

Walker reported on the root anatomy of maxillary first premolars, mandibular first premolars and the high incidence of three- rooted mandibular first molars in Asian patients. He did not however, report on the incidence of a second mesiobuccal canal (MB2) in the maxillary first molar [9]. Ratanajirasut et al. reported three separated roots of maxillary first molar in $99.8 \%$ of cases in Thai population, and explained that MB2 canals in the MB roots were present in $80.93 \%$ and $82.59 \%$ of the first and second molars, respectively [10].

Zheng et al conducted a study regarding the correlation between the prevalence of MB2 in different age groups.It was observed that the presence of MB2 was higher in young age group with $48.8 \%$ than in middle age group with $33.2 \%$ and elderly group with $18 \%$. Many studies concluded that the prevalence of MB2 decreases by aging due to dentine apposition which results in narrowing the MB2 canals [11]. Alrahabi et al conducted a study of root canal morphology of maxillary molars using cone beam computed tomography. They reported that majority of maxillary first molars (94\%) had three distinctly separated roots and 6\% had four roots. Palatal and distobuccal roots were observed to contain one root canal $(100 \%)$ and Vertucci's type I configuration. The mesiobuccal root had one $(29.4 \%$; type I) or two canals $(70.6 \%$; type II, III or IV) [12].

Walker conducted a study on Root form and canal anatomy of mandibular first molars in a southern Chinese population. Hereported $15 \%$ of the mandibular first molars examined were found to be 3 rooted, $45 \%$ of the sample displayed two distal canals and $28 \%$ of the teeth had 2 separate distal apical foramina [13]. Weine et al conducted a study on Canal configuration of the mesiobuccal root of the maxillary first molar of a Japanese subpopulation. They reported that out of 293 teeth, 123 (42.0\%) were Type II, $89(30.4 \%)$ showed Type III systems and 10 (3.4\%) were Type IV. Suggestions for identification and treatment of the second canal in the MB root were presented in their study [14]. With a rich case bank, we have published extensive studies on the root canal morphology of maxillary first molars in the past decade [15, 
22]. However further studies using larger samples of subjects in different ethnic groups may reveal variations and precise details of the root canal morphology, which will be very useful to the clinicians.

\section{Conclusion}

According to our study, incidence of MB2 canals was $88.9 \%$. Out of 18 teeth with intact roots, 16 teeth had MB2 canals. Type II Vertucci classification was seen in most of the teeth, where MB2 canals were present. Clinicians should always presume the presence of MB2 canals during the endodontic procedures to ensure that the most effective treatment results.Conventional radiographs usually do not produce the fact of existing MB2 canals. Thus, CBCT is the gold standard diagnostic tool in complex and in retreatment of missed root canals.

\section{References}

[1]. Cleghorn BM, Christie WH, Dong CC. Root and root canal morphology of the human permanent maxillary first molar: a literature review. J Endod. 2006 Sep;32(9):813-21. Pubmed PMID: 16934622.

[2]. Martins JNR, Marques D, Silva EJNL, Caramês J, Mata A, Versiani MA. Second mesiobuccal root canal in maxillary molars-A systematic review and meta-analysis of prevalence studies using cone beam computed tomography. Arch Oral Biol. 2020 May;113:104589. Pubmed PMID: 31735252.

[3]. Neelakantan P, Subbarao C, Ahuja R, Subbarao CV, Gutmann JL. Conebeam computed tomography study of root and canal morphology of maxillary first and second molars in an Indian population. J Endod. 2010 Oct;36(10):1622-7. Pubmed PMID: 20850665.

[4]. Silva EJ, Nejaim Y, Silva AI, Haiter-Neto F, Zaia AA, Cohenca N. Evaluation of root canal configuration of maxillary molars in a Brazilian population using cone-beam computed tomographic imaging: an in vivo study. J Endod. 2014 Feb;40(2):173-6. Pubmed PMID: 24461399.

[5]. Al-Shehri S, Al-Nazhan S, Shoukry S, Al-Shwaimi E, Al-Shemmery B. Root and canal configuration of the maxillary first molar in a Saudi subpopulation: A cone-beam computed tomography study. Saudi Endodontic Journal. 2017 May 1;7(2):69.

[6]. Vertucci FJ. Root canal morphology and its relationship to endodontic procedures. Endodontic topics. 2005 Mar;10(1):3-29.

[7]. Weine FS, Healey HJ, Gerstein H, Evanson L. Canal configuration in the mesiobuccal root of the maxillary first molar and its endodontic significance. Oral Surg Oral Med Oral Pathol. 1969 Sep;28(3):419-25. Pubmed
PMID: 5257186.

[8]. Vertucci FJ, Gegauff A. Root canal morphology of the maxillary first premolar. J Am Dent Assoc. 1979 Aug;99(2):194-8. Pubmed PMID: 287737.

[9]. Walker RT. Root form and canal anatomy of maxillary first premolars in a southern Chinese population. Endod Dent Traumatol. 1987 Jun;3(3):1304. Pubmed PMID: 3476301.

[10]. Ratanajirasut R, Panichuttra A, Panmekiate S. A Cone-beam Computed Tomographic Study of Root and Canal Morphology of Maxillary First and Second Permanent Molars in a Thai Population. J Endod. 2018 Jan;44(1):5661. Pubmed PMID: 29061352.

[11]. Zheng QH, Wang Y, Zhou XD, Wang Q, Zheng GN, Huang DM. A cone-beam computed tomography study of maxillary first permanent molar root and canal morphology in a Chinese population. J Endod. 2010 Sep;36(9):1480-4. Pubmed PMID: 20728713.

[12]. Alrahabi M, Sohail Zafar M. Evaluation of root canal morphology of maxillary molars using cone beam computed tomography. Pak J Med Sci. 2015 Mar-Apr;31(2):426-30. Pubmed PMID: 26101504.

[13]. Walker RT. Root form and canal anatomy of mandibular first molars in a southern Chinese population. Endod Dent Traumatol. 1988 Feb;4(1):1922. Pubmed PMID: 3268414.

[14]. Weine FS, Hayami S, Hata G, Toda T. Canal configuration of the mesiobuccal root of the maxillary first molar of a Japanese sub-population. Int Endod J. 1999 Mar;32(2):79-87. Pubmed PMID: 10371900.

[15]. Razumova S, Brago A, Khaskhanova L, Barakat H, Howijieh A. Evaluation of Anatomy and Root Canal Morphology of the Maxillary First Molar Using the Cone-Beam Computed Tomography among Residents of the Moscow Region. Contemp Clin Dent. 2018 Jun;9(Suppl 1):S133-S136. Pubmed PMID: 29962778.

[16]. Malay KK, Duraisamy R, Brundha MP, Kumar MP. Awareness regarding anemia among 1 st year dental undergraduate students. Drug Invention Today. 2018 Aug 1;10(8).

[17]. Kumar MS. Knowledge, attitude and practices towards oral health among law students in Chennai. Journal of Pharmaceutical Sciences and Research. 2016 Jul 1;8(7):650.

[18]. Kumar MP. Dental management of patients on antiplatelet therapy: Literature update. Asian J Pharm Clin Res. 2016;9(3):26-31.

[19]. Kumar S. Newer delivery systems for local anesthesia in dentistry. J Pharm Sci Res. 2015;7(5):252-5.

[20]. Ahamed A, Kumar MS. Knowledge, attitude and perceived confidence in handling medical emergencies among dental students. Journal of Pharmaceutical Sciences and Research. 2016 Jul 1;8(7):645.

[21]. Kumar S. Knowledge, attitude and practices of dental students toward dental management of patients on antiplatelet therapy. Asian J Pharm Clin Res. 2016;9(30):270-6.

[22]. Gayathri MM. Knowledge, Awareness and Attitude among dental students about hepatitis B infection. Journal of Pharmaceutical Sciences and Research. 2016 Mar 1;8(3):168. 